\title{
Estudios morfoanatómicos en Cattleya trianae (Orchi- daceae). I. Etapas tempranas del desarrollo del fruto
}

\author{
Miguel Ángel Gamboa-Gaitán
}

Laboratorio de Biología Tropical, Departamento de Biología, Universidad Nacional de Colombia, Carrera 30 № 45-03 Bogotá, D. C. Autor para correspondencia: magamboaga@unal.edu.co.

\section{Resumen}

La flor nacional de Colombia, Cattleya trianae, es un emblemático ejemplo de las orquídeas ornamentales de importancia económica. Si bien algunos estudios se han realizado en aspectos como reproducción y ecología, no hay estudios morfoanatómicos registrados para esta especie, por lo que con el presente manuscrito se pretende iniciar una serie de publicaciones que aporten información en este campo acerca de una especie particularmente atractiva por ser un símbolo nacional. Este estudio se centra en la anatomía del ovario y en los cambios que experimenta en los siguientes días a la polinización. El ovario muestra dos cavidades longitudinales, una para atraer al polinizador y otra para contener los óvulos que serán semillas tras la fertilización. Varios cambios anatómicos como la acumulación de un material de depósito que tapona el acceso a los óvulos y la formación de haces vasculares, son notables en los primeros días luego de la polinización en esta especie. La importancia de dichos cambios así como el uso de la anatomía para el estudio de la historia natural de las especies se discute aquí, ya que hay posiciones contradictorias al respecto.

Palabras clave: anatomía vegetal, desarrollo del fruto, ovario.

Editor: Hernández-Fernández J.

Citation: Gamboa-Gaitán MA. (2015). Estudios morfoanatómicos en Cattleya trianae (Orchidaceae). I. Etapas tempranas del desarrollo del fruto. Revista Mutis 5(1); pag 6-13.

Received: April 4, 2015; Accepted: May 23, 2015; Published on line: June $30,2015$.

Copyright: $\odot 2015$ Gamboa. This is an open-access article, which permits unrestricted use, distributions and reproduction in any medium, provided the original author and source are credited.

Competing Interests: The authors have no conflict of interest.
Morphoanatomic studies of Cattleya trianae (Orchidaceae). I. Early stages of fruit development

\begin{abstract}
Cattleya trianae is the most emblematic plant species of Colombia. Surprisingly, very few studies have been performed in this plant and many aspects of its natural history are unknown. This is the first of a series of papers that will cover morphological and anatomical aspects of this plant, in an attempt to better understand the biology of $C$. trianae. In this paper the anatomical changes in ovary following fertilization are shown and discussed by using common anatomical and staining techniques. This ovary shows to cavities, one devoted to attract pollinators and the other one
\end{abstract}


is used for storing ovules that will become seeds after fertilization. Two notable changes are observed in the ovary of $C$. trianae after pollination: the deposition of a material that blocks the cavity in which seeds develop and the arising and growing of vascular bundles. The significance of these findings are discussed as well as the relative importance of anatomical studies in the natural history of orchids.

Keywords: plant anatomy, fruit development, ovary.

\section{Introducción}

Entre las más de 30.000 especies que constituyen la familia Orchidaceae (Dressler 1981, Chase et al., 2003), las especies con importancia económica son casi todas ornamentales (Ortiz et al., 1982; Arditti, 1992), siendo la única excepción las especies comestibles del género Vanilla. Por su gran valor comercial y su fácil recolección, las poblaciones nativas han sido sujeto de una extracción indiscriminada de individuos que, asociada a la destrucción de sus hábitats naturales, tiene a muchas especies en peligro de extinción (Calderón, 2007). Entre las orquídeas colombianas uno de los principales grupos ornamentales es el género Cattleya, el cual representa el típico taxón que se ha visto duramente afectado por la excesiva recolección de plantas silvestres y la destrucción de su hábitat (Arango, 1972; Ospinam 1958; citados por Constantino, 1984). En efecto, de acuerdo con la Oficina de Enlace de la Secretaría Distrital de Ambiente encargada del control del tráfico ilegal de flora silvestre, el género Cattleya es uno de los grupos con mayor número de individuos incautados en Bogotá, D. C. (Ordóñez-Blanco, com. personal). El estado actual de este género podría describirse como el de un grupo que presenta una disminución drástica de sus poblaciones naturales y una larga lista de híbridos y variedades ornamentales.

Una forma de contribuir a la preservación de estas especies amenazadas, es estudiar y dilucidar su biología reproductiva, lo cual permitiría desarrollar programas de reproducción y reintroducción de individuos a sus hábitats nativos. Una búsqueda somera en las bases de datos muestra claramente que la mayoría de los estudios reproductivos se concentran en la biología de la polinización en el campo, mientras que los aspectos posteriores a la polinización, tales como el desarrollo del ovario, la fertilización de los óvulos, la viabilidad de las semillas, etc., son mucho más escasos (Gamboa-Gai- tán, en prep.). Los notables cambios morfoanatómicos que se presentan en el ovario luego de la polinización son un ejemplo de lo importante que son las etapas subsecuentes, pues ellas determinan la cantidad y calidad de semillas formadas, aspectos que tienen incidencia directa en el éxito reproductivo de la planta.

Los estudios anatómicos en orquídeas son pocos y se cree que menos del $0,5 \%$ de las especies han sido estudiadas al nivel de su anatomía vascular (St-Arnaud \& Barabé, 1989). Esto no deja de ser curioso, ya que los patrones vasculares, además de ser fundamentales para el correcto desarrollo del fruto, pueden ser usados en la clasificación de las orquídeas, tal como lo ha demostrado Garay (1972). Trabajando con el género Cypripedium, St-Arnaud \& Barabé (1989) encontraron una impactante variación en la organización de la anatomía vascular de especies con características comunes, resaltando la importancia de estos aspectos anatómicos en la clasificación de las especies, especialmente si se estudian asociados a procesos reproductivos. También en Cypripedium (Liu et al., 2012) han hecho estudios sobre la dinámica del fruto en sus primeras etapas, pero más dirigidos a entender la biología reproductiva que la sistemática. Se han realizado estudios anatómicos dirigidos a clarificar aspectos taxonómicos en la tribu Vanilleae (Stern \& Judd, 2000). Sin embargo, un estudio realizado por Stern \& Carlsward (2006) en la subtribu Oncidiinae, muestra que el solo estudio de los caracteres anatómicos de partes somáticas no son útiles en taxonomía. En contraposición a este enunciado está la postura de Aybeke et al. (2010), quienes realzan la importancia de los estudios anatómicos detallados en la taxonomía de las orquídeas.

En el presente reporte se muestran los cambios anatómicos experimentados por el ovario de la "flor de mayo" (Cattleya trianae Linden \& Rchb.f.) en las primeras etapas luego de la polinización y se discuten las posibles implicaciones de estos cambios en la historia natural de la planta.

\section{Materiales y métodos}

Especie: Cattleya trianae es una planta de amplio uso ornamental y junto a otras nueve especies constituye el registro actual para Colombia (Constantino, 1984; Ortíz-Valdivieso, 2010). Esta riqueza, sin embargo, podría verse alterada ya que recientes estudios moleculares cambian la clasificación de las especies de Cattle- 
ya (Van den Berg, 2014). Por ser nativa de dicho país, poseer los colores usados en los símbolos patrios y estar dedicada al botánico colombiano más famoso (José Jerónimo Triana), es considerada la flor nacional de Colombia y es comúnmente llamada flor de mayo (Díaz-Piedrahita, 2001). En esta especie es común encontrar muchas variedades e híbridos, ya que es una planta muy apreciada por los coleccionistas. Por esta misma razón hay una fuerte presión extractiva de individuos de sus poblaciones nativas (particularmente en Cundinamarca, Huila y Tolima), y aunque numerosos individuos sobreviven en colecciones privadas, puede considerarse una especie amenazada (Calderón, 2007).

Muestreo: se escogieron cinco individuos de una colección privada de $C$. trianae y se autopolinizaron manualmente tres flores en cada planta. Un ovario de cada planta fue recolectado 5,10 y 20 días luego de la polinización (DLP). Los ovarios fueron divididos en tres segmentos y se pusieron $24 \mathrm{~h}$ en una solución fijadora (CRAFT III) (Curtis, 1986). Luego se lavaron en agua corriente y se deshidrataron en secuencias de n-propanol en los siguientes porcentajes: $50,70,80$, $90,95,100$ y $100 \%$ durante períodos de $4-12$ h. Se sumergieron en xilol al $100 \%$ dos veces y por último se montaron en bloques de parafina y se realizaron cortes de $6 \mu \mathrm{m}$ de espesor con un micrótomo de deslizamiento manual. Los cortes se tiñeron con safranina (24 h) y verde rápido durante $15 \mathrm{~min}$, con un paso previo de desparafinación en xilol (15 h) y uno intermedio de limpieza en n-propanol $100 \%$. Posteriormente se hicieron montajes permanentes en laminillas portaobjetos de vidrio, los cuales se observaron e interpretaron al microscopio óptico marca Leica ${ }^{\circledR}$.

\section{Resultados}

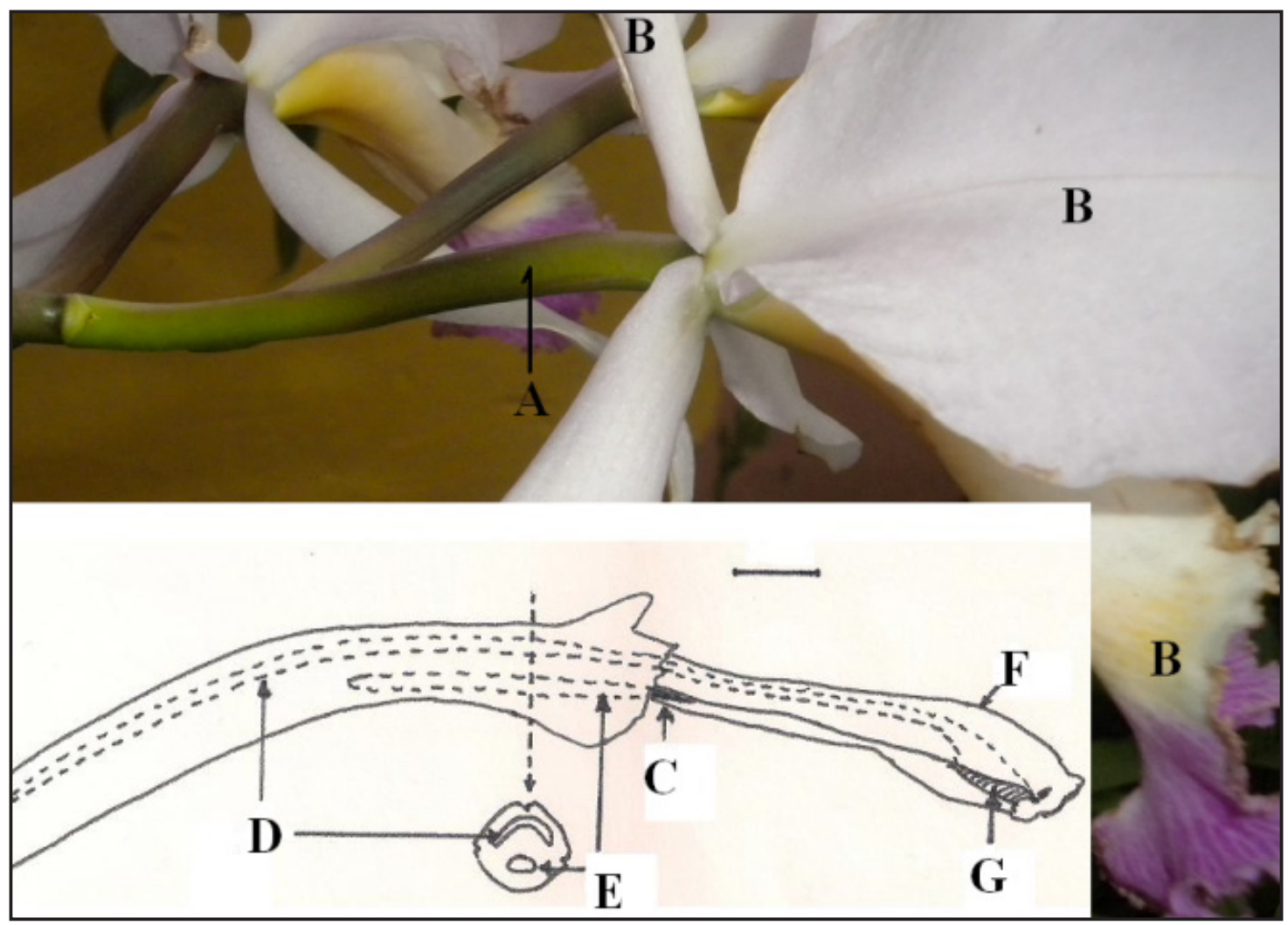

Figura 1. Vista lateral de un ovario no fecundado de $C$. trianae. A) Ovario ínfero, B) tépalos. En el esquema inferior se ha retirado el perianto y se esquematiza parte del interior de las estructuras reproductivas, C) entrada al canal nectarífero, D) canal ovárico, E) canal nectarífero, F) columna, G) estigma. 
El análisis anatómico del ovario no fecundado de $C$. trianae (figura 1), muestra en su primer tercio un canal ovárico obliterado, que posee un enmallado en toda su superficie interna. Este enmallado presenta una hebra más gruesa, casi central, que parece constituir una especie de eje. También se aprecia el canal nectarífero, semiovalado, con un amplio lumen que se abre en la base de la columna y penetra el ovario aproximadamente un tercio de su longitud total. Dicho canal está tapizado por un epitelio de células piramidales, posiblemente con función secretora (figura
2B), seguido de dos o tres capas de células aplanadas y de paredes más gruesas que las del tejido circundante. Se aprecian paquetes vasculares dispersos de pequeño tamaño (figuras 2 y 3 ). A este mismo nivel en un ovario de cinco DLP, existe una disposición igual de haces vasculares pero de mayor tamaño, sin embargo el canal nectarífero conserva su forma y tamaño. En algunos puntos el enmallado del canal ovárico está roto y en otros sitios se ha desprendido de la pared. El perímetro del ovario se torna ligeramente ondulado.

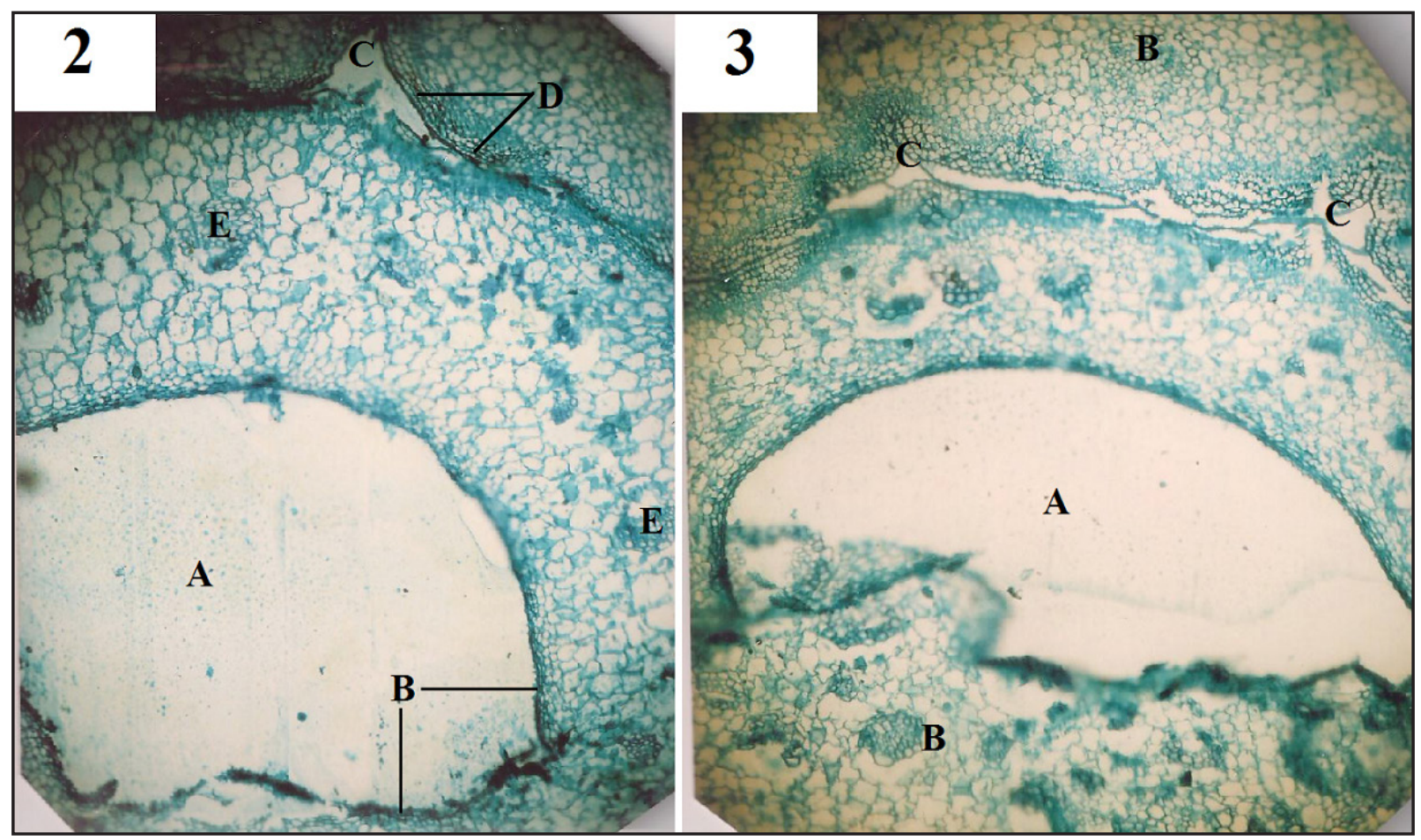

Figura 2. Corte transversal de ovario de $C$. trianae en el tercio distal. A) Canal nectarífero, B) epitelio secretor, C) canal ovárico, D) malla en el lumen del canal ovárico, E) haces vasculares.

Tras diez DLP el perímetro presenta ondulaciones amplias y en los lugares por donde se efectuará la dehiscencia del fruto (unión de los carpelos), se observan haces vasculares. Los haces vasculares del estroma se han agrandado y el enmallado del canal ovárico se diferencia poco (figura 4). Luego de 20 DLP se presen-
Figura 3. Corte transversal en el tercio distal del ovario de $C$. trianae 5 días luego de la polinización. A) Canal nectarífero, B) haces vasculares, C) enmallado del canal ovárico, obsérvese el desprendimiento.

ta un tapón de naturaleza fibrilar a lo largo del canal ovárico, que abarca desde la columna (figuras 5 y 6) hasta el primer tercio del ovario (figura 7). En esta etapa los haces vasculares son mucho más grandes y el ovario ha duplicado o incluso triplicado su tamaño. 


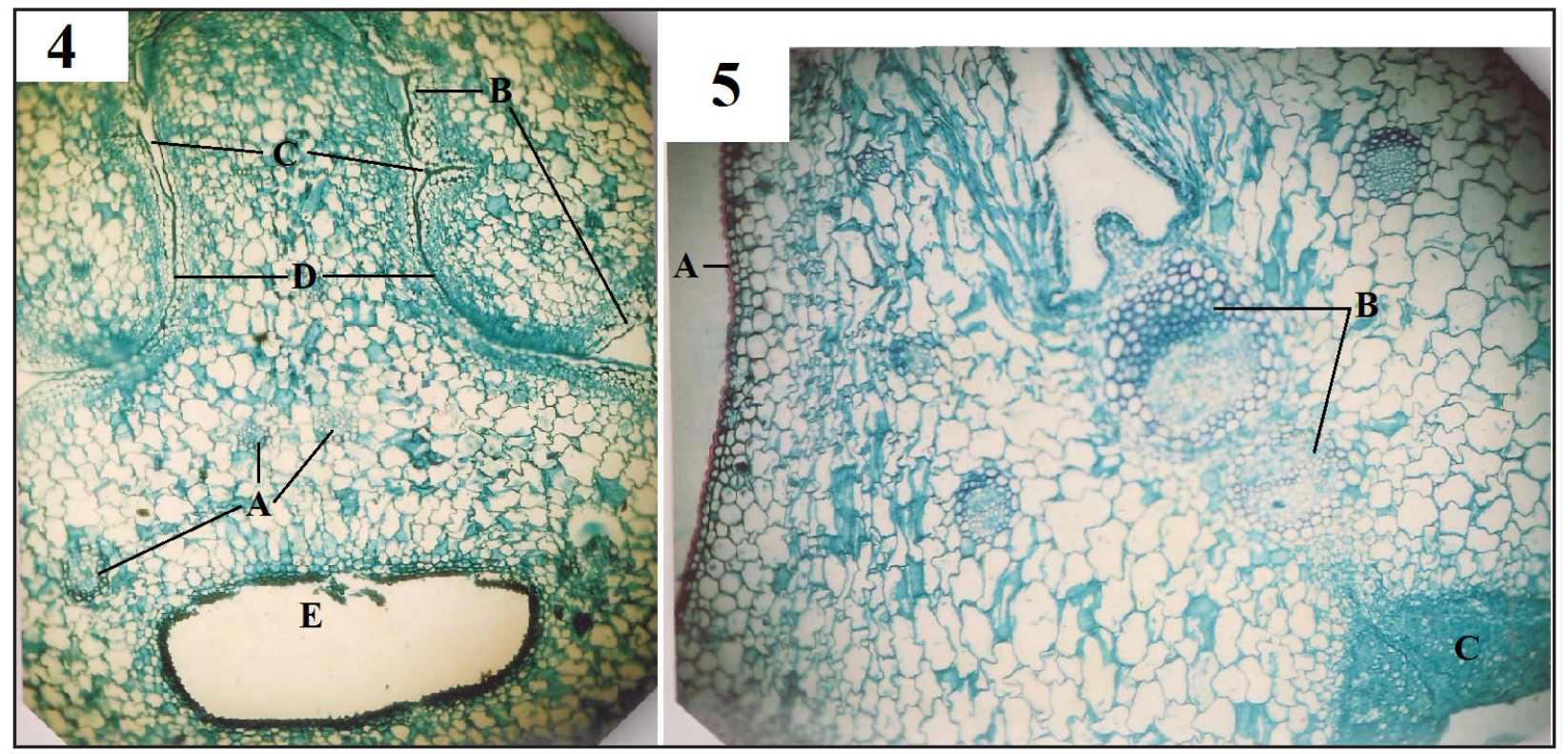

Figura 4. Corte transversal en el tercio distal del ovario de C. trianae 10 días luego de la polinización. A) Haces vasculares, B) malla del canal ovárico que se encuentra completamente obliterado, C) desprendimiento de la pared, D) hebra central, E) canal nectarífero.
Figura 5. Corte transversal en zona de dehiscencia del ovario de $C$. trianae 20 días luego de la polinización. A) Cutícula, B) haz vascular sobre la línea de dehiscencia, C) enmallado cubierto de material de depósito en el canal ovárico.

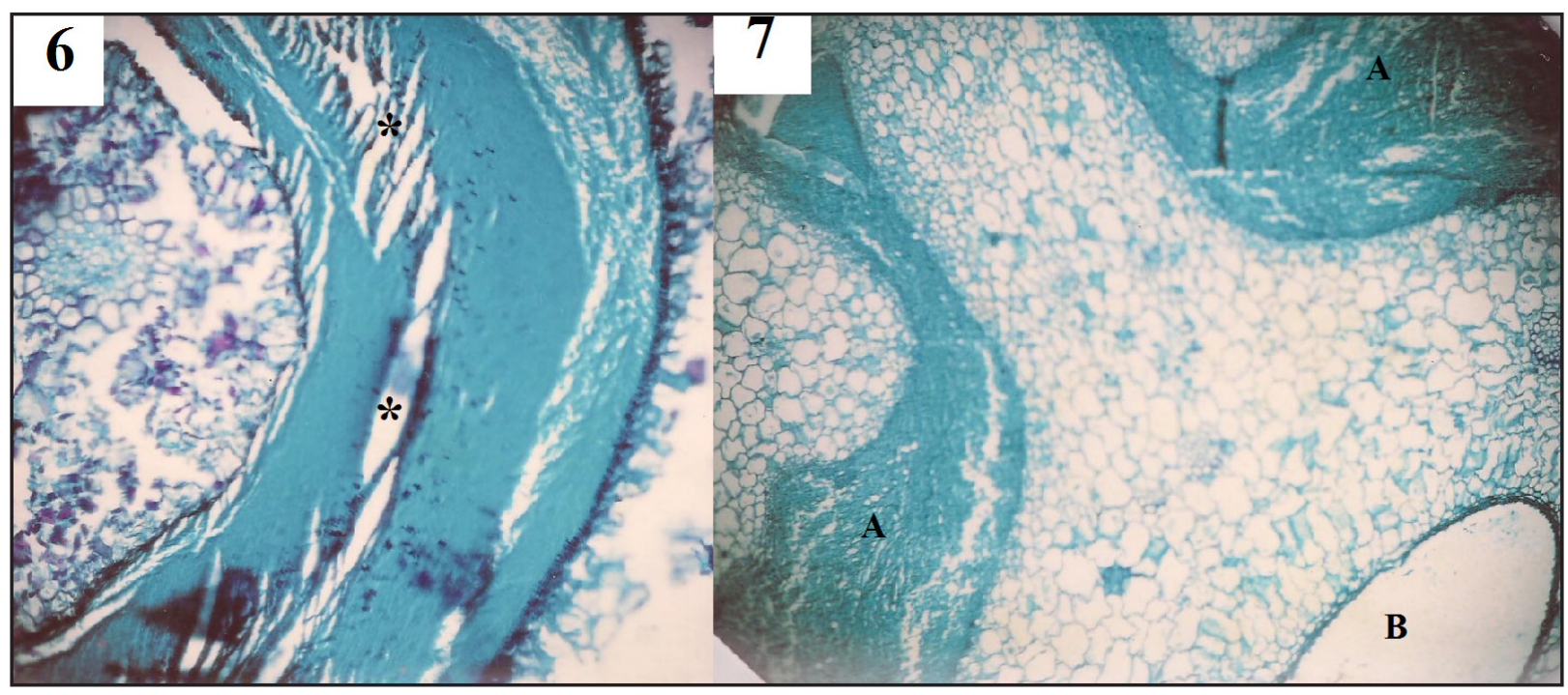

Figura 6. Corte transversal del canal ovárico de $C$. trianae a nivel de columna 20 días luego de la polinización. Se evidencia en detalle el material de depósito que se organiza en torno al enmallado que se encuentra en su interior. Nótese su naturaleza no celular, los espacios en su interior son probablemente artefactos producidos por el desgarro del tejido durante el montaje $(*)$.
Figura 7. Corte transversal del ovario de $C$. trianae 20 días luego de la polinización. A) Canal ovárico completamente lleno de material de depósito, B) canal nectarífero dispuesto basalmente en la flor normal. 
En el tercio medio del ovario fecundado se observa un lóculo con pliegues gruesos de los cuales parecen desprenderse unas masas celulares compactas (figura 8), oscuras, con núcleo bien definido y de diversa ubicación en la célula. El parénquima que rodea al lóculo hace transición con este a través de un tejido circular de células aplanadas, dispuestas en unas 4-8 capas. En los amplios pliegues de la pared del lóculo se depositan cristales.

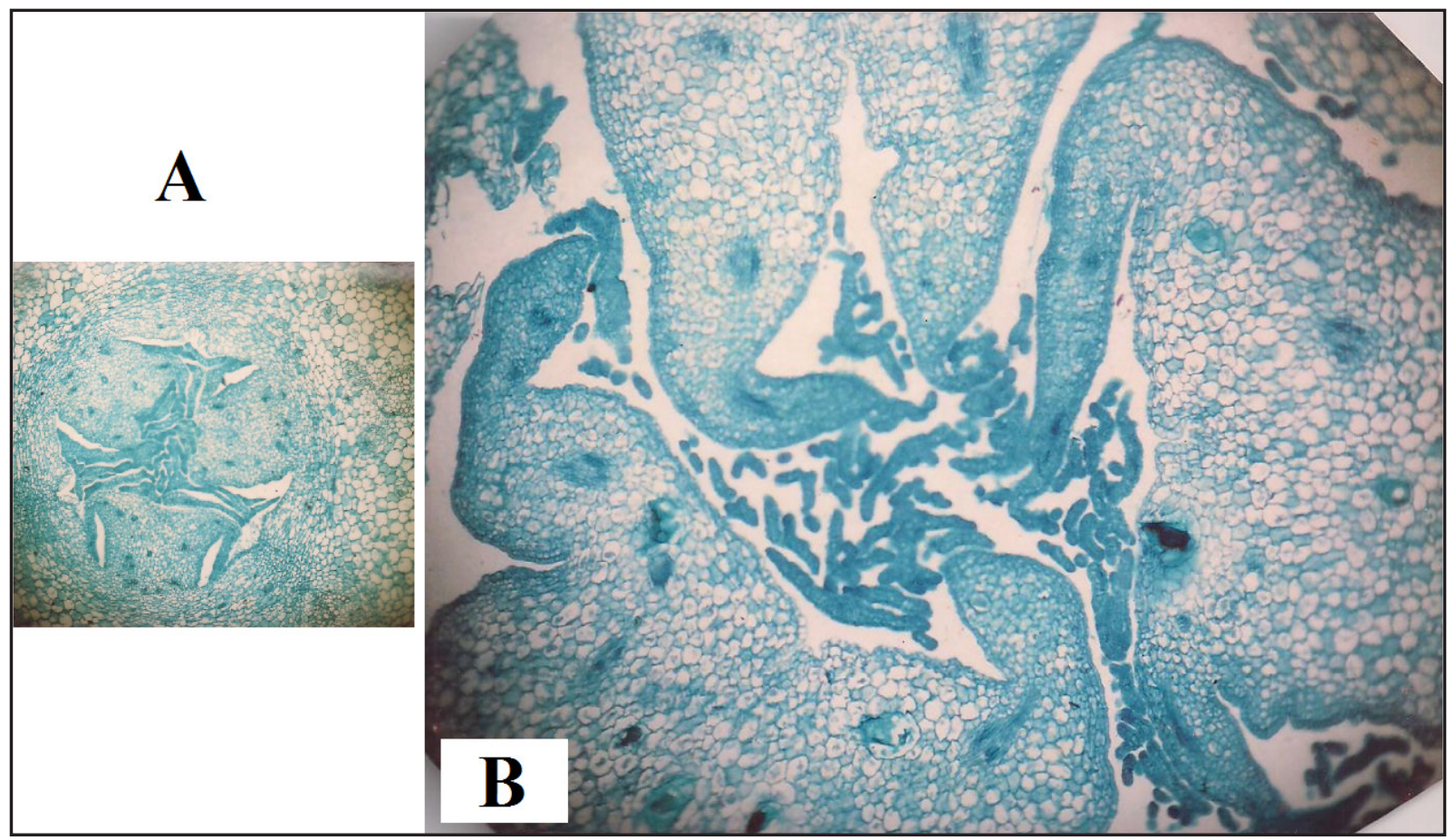

Figura 8. Corte transversal del tercio medio del ovario de $C$. trianae 20 días luego de la polinización. A) Se aprecian acúmulos de naturaleza celular en la luz del lóculo, B) vista aumentada para mostrar los acúmulos que parecen ser semillas en formación. Son notables los pliegues de la pared del lóculo que aumentan la superficie disponible.

\section{Discusión}

Notables cambios anatómicos se verifican en el ovario de $C$. trianae poco después de la polinización, e incluyen el aumento del tamaño de los haces vasculares, la formación de haces vasculares sobre los futuros sitios de dehiscencia y el depósito de un material fibroso rellenando la columna y el tercio distal del canal ovárico.

En estado maduro, el fruto de $C$. trianae es una cápsula de aproximadamente $10 \mathrm{~cm}$ de longitud por 3,5 $\mathrm{cm}$ de ancho, cuya dehiscencia se verifica entre seis y nueve meses luego de la polinización. El tener que conservar un fruto tan grande durante tanto tiempo, implica grandes gastos para el individuo ya que en las orquídeas es necesario realizar una producción masiva de semillas debido a la baja probabilidad de sobrevivencia de las plántulas y la escasez de polinizaciones exitosas (por ejemplo Calvo, 1993).
La producción del tapón que obstruye la entrada al canal ovárico, puede interpretarse como la estrategia usada ante la necesidad de mantener las semillas en formación en un ambiente estéril y a salvo de depredadores y patógenos. La composición de ese material se desconoce, pero es viable pensar que sus componentes básicos sean carbohidratos, sustancias comúnmente usadas por las plantas en estas circunstancias. El compuesto más comúnmente asociado a esta función taponadora es la calosa, un polisacárido común en plantas, que es considerada una defensa polifacética de las plantas (Luna et al., 2011), así que pruebas para la detección de este compuesto deberán ser realizadas en primera instancia en la siguiente fase de este proyecto. Es viable postular que el enmallado presente en los ovarios no polinizados sirve de base estructural para la posterior acumulación del material de relleno. 
Los desgarros observados en el enmallado no pueden atribuirse al paso de tubos polínicos, pues fue común observar en varias clases de tejidos estos desprendimientos. Al parecer fue el arrastre de la cuchilla del micrótomo el causante de ellos, por lo que habría que mejorar la calidad de la fijación del material vegetal. Es muy probable que las técnicas aquí empleadas impidan la observación de los tubos polínicos. De haberse originado un tubo por cada semilla formada, cientos de miles de tubos debieron haber sido observados, pues en este género se producen hasta un millón de semillas. Las masas celulares compactas del tercio medio del ovario (figura 8), tienen cierta semejanza con lo reportado por Mayer et al. (2011), que ellos atribuyen a la presencia de un embrión con un suspensor bien desarrollado.

Se postula, con base en los datos obtenidos, que la polinización en $C$. trianae es seguida por una migración masiva de granos de polen (o sus tubos polínicos) hasta la parte medial del ovario, donde se efectúa la fecundación de los óvulos e inmediatamente después se forma un tapón que impide el acceso al banco de semillas en formación. Dicho tapón se establece sobre el enmallado fibrilar preexistente en el canal ovárico que le sirve de matriz basal. Se observa también un incremento en el número y tamaño de los haces vasculares del fruto, lo cual es compatible con la necesidad de nutrir miles de semillas en formación.

Es necesario continuar la investigación en aspectos como la composición química y el origen del material de depósito, la detección y migración de los núcleos espermáticos desde los granos de polen en el estigma hasta los óvulos y la naturaleza de las interacciones fisiológicas de los tubos polínicos con su entorno ovárico.

\section{Referencias}

Arango, C. (1972). Cattleyas Colombianas. VII Conferencia Mundial de Orquideología, Programa Commemorativo.

Arditti, J. (1992). Fundamentals of orchid biology. John Wiley \& Sons, Nueva York.

Aybeke, M., Sezik, E., \& Olgun, G. (2010). Vegetative anatomy of Ophrys, Orchis, and Dactylorhiza (Orchidaceae) taxa in Trakya región of Turkey. Flora 205: 73-89.
Calderón, E. (2007). Libro rojo de plantas de Colombia. Volumen 6, primera parte. Orquídeas. Instituto de Investigación de Recursos Biológicos Alexander von Humboldt, Bogotá, D. C.

Calvo, R. N. (1993). Evolutionary Demography of orchids: Intensity and frequency of pollination and the cost of fruiting. Ecology 74(4): 1033-1042.

Chase, M., Freudenstein, J., Cameron, K., \& Barrett, R. (2003). DNA data and Orchidaceae systematics: a new phylogenetic classification. En Dixon, K., Kell, S., Barrett, R., \& Cribb, P. (Eds.). Orchid Conservation. Natural History Publications. (págs. 69-89). Kota Kinabalu, Malasia.

Constantino, E. (1984). Las Orquídeas del Género Cattleya en Colombia. Fundación Herencia Verde, División de Publicaciones, Santiago de Cali.

Curtis, J. (1986). Microtecnia Vegetal. Editorial Trillas, S. A., México D. F.

Díaz-Piedrahita, S. (2001). La Flor de Mayo, Cattleya trianae, flor nacional. Revista Credencial Historia, edición 139.

Dressler, R. L. (1981). The Orchids: natural history and classification. Harvard University Press, Cambridge.

Garay, L. A. (1972). On the Origin of the Orchidaceae, II. Journal of the Arnold Arboretum 53: 202-215.

Liu, F., Tian, M., Wang, C. X., Gong, M. J. \& Li, Q. J. (2012) Observation on fruit growth dynamics and embryo development process of Cypripedium japonicum. Journal of Plant Research and Environment 21: 28-35.

Luna, E., Pastor, V., Robert, J., Flors, V., Mauch-Mani, B., \& Ton, J. (2011). Callose deposition: a multifaceted plant defense response. Molecular Plant Microbe Interactions 24(2): 183-93.

Mayer, J. L. S., Carmello-Guerreiro, S. M., \& Appezzato-da-Glória, B. (2011). Anatomical development of the pericarp and seed of Oncidium flexuosum Sims (Orchidaceae). Flora 206(6): 601-609.

Ortiz-Valdivieso, P. (2010). Orquídeas: Especies de Colombia. Villegas Editores, Bogotá, D. C. 
Ortiz, P., Martínez, \& M. A., Misas, G. (1982). Orquídeas Ornamentales de Colombia. Carlos Valencia Editores, Santafé de Bogotá.

Ospina, M. (1958). Orquídeas Colombianas. Publicaciones Técnicas Ltda., Santafé de Bogotá.

ST-Arnaud, M., \& Barabé, D. (1989). Comparative analysis of the flower vascularization of some Cypripedium species (Orchidaceae). Lindleyiana 4(3): 146-153.

Stern, W. L., \& Carlsward, B. S. (2006). Comparative vegetative anatomy and systematics of the Onci- diinae (Maxillarieae, Orchidaceae). Botanical Journal of the Linnean Society 152(1): 91-107.

Stern, W. L., \& Judd, W. S. (2000). Comparative anatomy and systematics of the orchid tribe Vanilleae excluding Vanilla. Botanical Journal of the Linnean Society 134(1-2): 179-202.

Van den Berg, C. (2014). Reaching a compromise between conflicting nuclear and plastid phylogenetic trees: a new classification for the genus Cattleya (Epidendreae; Epidendroideae; Orchidaceae). Phytotaxa, 186(2):75-86. 